\title{
Análisis de los procesos de uso de artefactos líticos en sociedades cazadoras-recolectoras. Ocupaciones correspondientes a la transición Pleistoceno/ Holoceno, meseta central de Santa Cruz
}

\author{
( Manuel E. Cueto* \\ Fecha de defensa: 18/11/2014 \\ Directora: Dra. Alicia S. Castro \\ Jurados: Dres. J. Bautista Belardi, Rafael Goñi \\ y Laura Miotti.
}

\section{Acerca del problema de investigación}

En mi trabajo de Tesis Doctoral realicé un estudio de la tecnología lítica dirigido a abordar el uso de los artefactos, el modo de empleo, la relación forma-función y la distribución espacial de actividades dentro de un grupo de sitios arqueológicos. Con este propósito se efectuó el análisis de conjuntos artefactuales de dos momentos de ocupación consecutivos, el Pleistoceno Final (13.000-10.0000 AP) y el Holoceno Temprano (10.000-7500 AP), de sitios reparados del ámbito de la Meseta central de Santa Cruz, Patagonia Argentina. Los sitios seleccionados para el estudio fueron Casa del Minero 1 (CDM1), Cueva Túnel (CT), Cueva de la Ventana (LV) y Cueva La Mesada (LM) que se ubican en la Localidad Arqueológica La María distante a $150 \mathrm{~km}$ al noroeste de la ciudad de Puerto San Julián. Esta localidad destaca por su alta visibilidad arqueológica, con numerosos sitios en cuevas y reparos rocosos, sectores de acampe a cielo abierto, fuentes de materia prima lítica con signos de explotación, y gran cantidad de pinturas rupestres (Paunero et al. 2005). Las investigaciones en La María se inician en la década de 1980 bajo la dirección del Ing. Augusto Cardich y luego se desarrollan por el Lic. Rafael Paunero y equipo, quienes despliegan diversas líneas de investigación en el marco del proyecto "Investigaciones arqueológicas en la Meseta Central de Santa Cruz, Pasado Humano y Comunicación" (Cueto et al. 2014). En este contexto el objetivo principal de mi Tesis apuntó a contribuir al conocimiento de la tecnología lítica en relación a las estrategias de producción y consumo que implementaron las primeras sociedades cazadoras recolectoras que habitaron la meseta. A su vez se atendió un conjunto de objetivos específicos como el estudio de huellas de utilización a partir de colecciones experimentales; el examen de las secuencias de producción de instrumentos con énfasis en la etapa de formatización

CONICET - División Científica de Arqueología, FCNyM, UNLP. Edificio Anexo, Laboratorio 107, PB (CP1900), La Plata, Buenos Aires, Argentina. Email: manuelcueto@fcnym.unlp.edu.ar final y las características morfo-funcionales de las porciones activas; y, por último, la identificación de similitudes y diferencias en las técnicas de manufactura y uso de artefactos entre los conjuntos de ambos momentos.

El arribo de los primeros grupos a Patagonia ocurrió durante el Pleistoceno final, período de clima frío y paisaje árido atravesado por granes fluctuaciones ambientales; habría sido un proceso de poblamiento caracterizado por una demografía muy baja. Para la Meseta central se propone un modelo de movilidad residencial restringida debido a la existencia de áreas donde se concentraban los recursos. Los grupos habrían desplegado una estrategia de subsistencia generalísta de caza del guanaco con uso oportunístico de megamamíferos (Miotti y Salemme 2004). El deshielo y la disminución de las características de continentalidad durante el Holoceno temprano aumentan las posibilidades de expansión territorial y formación de redes sociales en un contexto de incremento poblacional. No obstante en algunos sectores el agua pudo volverse un recurso crítico debido a las bajas precipitaciones (Borrero 1999) resultando espacios menos atractivos. En este sentido Paunero (2009) sugiere que en la meseta se habría dado una posible disminución de la ocupación humana en los primeros mil años del Holoceno, y un incremento a partir del 9000 ${ }_{14}$ C AP. Se postula que los grupos habrían desarrollado una estrategia de movilidad logística con restructuración de espacios, definición de áreas específicas de actividad, redundancia en el uso de lugares y mejor conocimiento del ambiente. Pudo haber continuidad respecto de las ocupaciones pleistocénicas en la estrategia de subsistencia, aunque otros proponen un cambio hacia el consumo casi especializado del guanaco (Borrero 1999; Miotti y Salemme 2004). Además de las dinámicas de poblamiento y en pos de lograr una discusión exhaustiva de las estrategias de producción-consumo, se consideraron los patrones tecnológicos y funcionales alcanzados en el estudio de sitios contemporáneos de la región como Cerro Tres Tetas 1; Los Toldos 3; Piedra Museo; El Ceibo 7; La Martita y El Verano (Paunero 2009).

\section{Marco teórico-metodológico}

En cuanto a los lineamientos teóricos, utilicé un concepto dinámico de tecnología que resulta de la articulación de 
la teoría de las prácticas sociales y la organización de la tecnología lítica. Así la tecnología es entendida como un conjunto de conocimientos y procedimientos materiales intencionales que mediante la implementación de gestos y operaciones permiten la explotación de recursos como la realización de un producto (Álvarez 2003). La misma no implica sólo los objetos y medios usados por la sociedad para actuar sobre el ambiente físico, sino que involucra todos los aspectos posibles del proceso de acción sobre la materia. Esta concepción trasciende al objeto material finito entendiendo el fenómeno tecnológico como un hecho cultural transmisible intergeneracionalmente y comprensible en su contexto socio-histórico particular (Lemmonier 1993). Siguiendo esta perspectiva, la producción es el proceso por el cual los miembros de una sociedad generan artefactos para satisfacer necesidades sociales mediante el uso de acuerdo con las características de su tecnología. Así el instrumento es el punto relacional entre ambos fenómenos dado que es la culminación de un proceso productivo y al mismo tiempo es un implemento que será utilizado en otra actividad (Briz 2006-2007). En consecuencia, a toda operación de trabajo realizada por un sujeto que involucra la manipulación de un artefacto lítico se la entiende por estrategia de consumo. Por medio de estos objetos los individuos extienden su capacidad de operar sobre el mundo con fines que pueden variar a través del tiempo (Ingold 1997). El factor espacial también genera variabilidad y en ocasiones pueden distinguirse patrones de distribución de restos, intra e intersitio, que dan cuenta de la organización espacial de las actividades y el patrón de ocupación de un sitio (Odell 2001). En diversos grupos existe preferencia por algunas litologías destinadas a la confección de instrumentos relacionados con trabajos específicos. A su vez se han detectado rasgos en los artefactos que responden a factores estilísticos, a partir de la determinación de funciones similares en instrumentos con morfologías diferentes. También se registra la producción de algunas clases de artefactos con baja inversión de trabajo que cumplieron múltiples funciones (Álvarez 2003; Mansur 1983).

La metodología implementada contempló cuatro análisis principales. Para el estudio de la funcionalidad de artefactos líticos apliqué el análisis funcional de base microscópica, considerando las propuestas de Mansur (1983), Castro (1994) y Álvarez (2003). Para clasificar los conjuntos líticos emplee una perspectiva que integra criterios tecnológicos desarrollados en las investigaciones en la Meseta central (Cueto et al. 2014) con elementos de la propuesta de Aschero (1975). Realicé una clasificación morfológica y tecnológica en base a un set de caracteres relevantes para el examen funcional (ángulo, longitud y delineación del filo). Además ordene los artefactos de cada litología según estadíos de reducción en base al modelo de Collins (1989-1990): 1 . preparación y reducción inicial del núcleo, 2. talla y extracción de formas base, y 3 . formatización final de artefactos.
A continuación integré los enfoques tecnomorfológico y funcional para determinar las estrategias de consumo que caracterizan a cada conjunto. Evalué el grado de integridad funcional de los artefactos por medio del análisis de correspondencia entre elementos del diseño artefactual (caracteres de las porciones activas y el tipo de roca) y las operaciones de trabajo según sustancia procesada y tipo de movimiento. Este análisis permite evaluar las distintas elecciones realizadas por el grupo en la producción de artefactos (Álvarez 2003). Por último evalué la estrategia de uso del espacio intrasitio para cada ocupación, considerando la información referente a las estrategias de producción y consumo de los artefactos de manera articulada a un enfoque distribucional (Odell 2001).

\section{Principales resultados}

En relación con las estrategias de producción de artefactos líticos se observa que tanto en las ocupaciones finipleistocénicas como del Holoceno temprano principalmente se explotaron rocas locales con predominio del sílex. Esto se vincula a la muy buena calidad para la talla de estas litologías, a su inmediata disponibilidad y a que resultan efectivas para el procesamiento de recursos. Además dentro de las cuevas se realizaron las etapas finales de la manufactura de artefactos. Estos últimos se caracterizan por el trabajo unifacial a partir de la selección de soportes no estandarizados, de módulo grande y manufacturados siguiendo secuencias con baja inversión de trabajo. Estas elecciones se vincularían, entre otros factores, con la adecuación a la prensión manual de los instrumentos como único modo de sujeción identificado. Dichas tendencias se inscriben de manera concordante con las registradas a nivel regional. Los grupos que ocuparon la localidad en ambos momentos también comparten prácticas a nivel de las estrategias de consumo. Se registra que los filos retocados o naturales y las superficies activas utilizadas fueron seleccionados para ejecutar una sola acción sobre una única sustancia. A su vez existe una preferencia por el uso de filos con formatización secundaria, siendo poco habitual el empleo de bordes naturales de productos de talla. Estos últimos se usaron principalmente en ocupaciones de actividades múltiples posiblemente como estrategia expeditiva en respuesta a necesidades inmediatas. Se trata de piezas con ángulo agudo utilizadas en acciones de corte de diversas sustancias, tendencia semejante a nivel regional. Entre las preferencias tecnológicas de ambos momentos predominan diseños con alto grado de especificidad funcional. Sin embargo las clases con mayor frecuencia entre los artefactos con uso, lasca retocada (Pleistoceno) y raedera (Holoceno), constituyen diseños versátiles con baja o nula especificidad funcional. Asimismo, el análisis funcional permitió identificar el aprovechamiento de sustancias que generalmente no se preservan en el registro (cuero, madera y carne) y ocuparon un rol importante en la 
alimentación y la manufactura de bienes. Aunque se explotó la misma variedad de recursos en ambos momentos, se observa un aumento en la explotación de la madera durante el Holoceno y una práctica mas frecuente de desposte y trozamiento de presas durante las ocupaciones iniciales. En síntesis, esta tesis permitió profundizar la comprensión de las dinámicas de producción de artefactos y explotación de recursos respecto a los estudios tecnológicos que solo indagan sobre el aprovisionamiento y la manufactura. Las tendencias observadas permiten postular el predominio de continuidades a nivel de las preferencias tecnológicas de uso y diseño de los filos por las sociedades cazadorasrecolectoras durante los primeros momentos de ocupación de la Meseta. Este hecho permite pensar más en la continuidad de los modos de hacer y de variadas prácticas culturales entre las sociedades que poblaron el área, que en un posible abandono por parte de los primeros pobladores y la consiguiente reocupación por nuevos grupos que desconocieran la misma. Sin pretender abarcar todas las relaciones de forma y función, esta Tesis conforma un modelo de como aproximarnos a la variabilidad de uso de los instrumentos desde una perspectiva que trasciende la concepción de los artefactos como de naturaleza unívoca.

\section{Bibliografía}

»ÁLVAREZ, M. 2003. Organización Tecnológica en el Canal Beagle. El caso Túnel I (Tierra del Fuego, Argentina). Tesis de Doctorado, Facultad de Filosofía y Letras, Universidad Nacional de Buenos Aires. Ms.

»ASCHERO, C. A. 1975. Ensayo para una clasificación morfológica de artefactos líticos aplicado a estudios tipológicos-comparativos. Informe al CONICET. MS.

" BORRERO, L. 1999. Human dispersal and climatic conditions during Late Pleistocene times in Fuego-Patagonia. Quaternary International 53/54: 93-99.

》 BRIZ, I. 2006-2007. Piedras, dinámicas, producciones y consumos: propuesta desde la Dialéctica para el análisis de los conjuntos líticos. Krei 9: 27-46.

»CASTRO, A. 1994. El análisis funcional de materiales líticos por medio de la observación microscópica de huellas de uso: un modelo alternativo de clasificación tipológica.
Tesis de Doctorado. Facultad de Ciencias Naturales, Universidad Nacional de La Plata.

» COLLINS, S. 1989-199o. Una propuesta conductual para el estudio de la arqueología lítica. Etnía 34-35: 47-65.

» CUETO, M., F. SKARBUN y A. FRANK. 2014. Tecnología lítica de los cazadores recolectores de la meseta central patagónica. Balances y perspectivas para una propuesta de integración. En Indústrias líticas na América do Sul: abordagens teóricas e metodológicas, editado por A. Lourdeau, S. Viana y M. Rodet, pp. 173-202. EdUFPE, Recife, Brasil.

» INGOLD, T. 1997. Eight themes in the Anthropology of technology. Techology as skilled practice. Social Analysis 41 (1): 106-138.

» LEMONNIER, P. 1993. Technological Choices: Transformation in Material Cultures Since the Neolithic. Routledge.

» MANSUR, M. E. 1983. Traces d' utilisation et technologie lithique: examples de la Patagonie. Thèse de zème cycle, Université de Bourdeaux I, Bordeaux.

» MIOTTI, L. y M. C. SALEMME. 2004. Poblamiento, movilidad y territorios entre las sociedades cazadoras-recolectoras de Patagonia. Complutum 15: 177-206.

» ODELL, G. 2001. Stone tool research at the end of the millennium: classification, function, and behavior. Journal of Archaeological Research 9: 45-100.

» PAUNERO, R. 2009. La colonización humana de la meseta central de Santa Cruz durante el Pleistoceno final: indicadores arqueológicos, referentes estratigráficos y nuevas evidencias. En Arqueología de Patagonia: una mirada desde el último confín, editado por M. C. Salemme, F. Santiago, M. Álvarez, E. Piana, M. Vazquez y M. E. Mansur, vol 1, pp. 85-100. Editorial Utopías, Ushuaia.

» PAUNERO, R., A. FRANK, F. SKARBUN, G. ROSALES, G. ZAPATA, M. CUETO, M. PAUNERO, D. MARTINEZ, R. LÓPEZ, N. LUNAZZI y M. DEL GIORGIO. 2005. Arte Rupestre en Estancia La María, Meseta Central de Santa Cruz: Sectorización y contextos arqueológicos. Relaciones de la Sociedad Argentina de Antropología XXX: 147-168. 